\title{
Grupos terapêuticos com profissionais da saúde durante a pandemia da covid-19: relato de experiência.
}

\section{Therapeutic groups with health professionals during the covid-19 pandemic: experience report.}

DOI: $10.46919 / \operatorname{archv2n3-015}$

Recebimento dos originais: 01/01/2021

Aceitação para publicação: 31/03/2021

\author{
Flávia Michelle Pereira Albuquerque \\ Mestre em Desenvolvimento e Políticas Públicas \\ Instituição: Universidade Federal da Fronteira Sul - UFFS \\ Endereço: Av santa Cruz 169 apto 3201 - Santa Rosa - RS - Brasil \\ E-mail: flaviampa@msn.com \\ Jocelene Francine Schons \\ Graduação em Psicologia \\ Instituição: SETREM - Sociedade Educacional de Três de Maio \\ Endereço: av Santa Rosa 2405 - Três de Maio - RS - Brasil \\ E-mail: joceschons20@gmail.com \\ Paula Roberta da Silva dos Santos \\ Graduanda em Psicologia \\ Instituição: SETREM - Sociedade Educacional de Três de Maio \\ Endereço: av Santa Rosa 2405 - Três de Maio - RS - Brasil \\ E-mail: paula.robt2@gmail.com
}

\begin{abstract}
RESUMO
O presente estudo refere-se a um relato de experiência desenvolvido com trabalhadores da área de saúde num Centro de Referência de Saúde do Trabalhador (CEREST). O objetivo do trabalho foi de discutir sobre os impactos e mudanças na saúde dos trabalhadores que estão atuando no enfrentamento a pandemia da COVID-19. Foram realizados grupos com os profissionais de saúde, homens e mulheres, com idade entre 20 e 60 anos. Com cada grupo foram realizados três encontros, no período de julho a setembro de 2020 . Por meio de dinâmicas grupais, foram levantadas questões relacionadas ao reflexo da pandemia sobre a saúde mental e estratégias de enfrentamento. Os impactos do Coronavírus na saúde dos trabalhadores ainda são desconhecidos. Os relatos apontam a necessidade de cuidados com os trabalhadores da saúde pública, sendo indispensável a identificação das necessidades, demandas e problemas de saúde dos trabalhadores, assim como garante a própria Política Nacional de Saúde do Trabalhador e da Trabalhadora. Entre as dificuldades relatadas, estão: sobrecarga de trabalho, medo, ansiedade, sentimentos de não pertencimento no grupo de trabalho. A experiência permitiu conhecer as peculiaridades de cada grupo, bem como, ampliar discussões sobre o tema, oferecendo subsídios para repensar o processo de trabalho.
\end{abstract}

Palavras-chaves: Saúde do trabalhador. Covid-19. Saúde mental. Psicologia.

\section{ABSTRACT}

The present study refers to an experience report developed with health workers from a Worker Health Reference Center (CEREST). The objective of the work was to discuss the impacts and changes in the health of workers who are acting in the control of the pandemic of COVID-19. Men and women, aged 
between 20 and 60, participated in the groups. Three meetings were held with each group from July to September 2020. Through group dynamics, issues related to the pandemic's reflection on mental health and coping strategies were raised. The impacts of Coronavirus on workers' health are still unknown. Reports point to the need for care of public health workers, and it is essential to identify the needs, demands, and health problems of workers, as well as to guarantee the National Worker and Worker Health Policy itself. Among the difficulties reported are: work overload, fear, anxiety, group health divergence. The experience allowed for knowing the peculiarities of each group, as well as expanding discussions on the topic, offering subsidies to rethink the work process.

Keywords: Workers' health. Covid-19. Mental health. Psychology.

\section{INTRODUÇÃOO}

O Brasil e o mundo atualmente têm enfrentado os efeitos advindos da COVID-19. Diversos aspectos da vida social e individual do sujeito sofreram fortes impactos em um curto período de tempo (MATTEI, 2020). A Organização Mundial de Saúde (OMS) em março de 2020 declarou estarmos enfrentando uma pandemia mundial, fazendo com que nações atentassem para a necessidade de pensar em estratégia para minimizar os danos à saúde da coletividade (SCHMIDT et al., 2020).

Neste cenário, emerge enquanto protagonistas no cuidado coletivo, os trabalhadores de saúde que estão na linha de frente prestando a assistência de maneira direta e indireta a toda a população (LIMA, 2020). Acrescido a isso cabe ressaltar que o trabalhador da área da saúde tem estado exposto a um cenário amplamente dinâmico quanto orientações técnicas, garantia de insumos, equipamentos e incertezas a êxitos das condutas terapêuticas (MAEYAMA et al., 2018).

A COVID-19, tornou o mundo do trabalho ainda mais complexo e multifacetado. Os acidentes de trabalho têm significativa morbimortalidade, consolidando-se como um grande problema de saúde pública. Conforme dados do Ministério do Trabalho e Emprego, no Brasil ocorrem cerca de 723 mil acidentes de trabalho por ano, causando a morte de 2.496 pessoas em acidentes de trabalho, ou seja, cerca de 7 trabalhadores por dia (BRASIL, 2020).

Por meio de Portaria 205/2016, o Ministério da Saúde tornou a notificação compulsória no Sistema de Informação de Notificação de Agravos do Sistema Único de Saúde (SINAN), dos agravos à saúde do trabalhador, incluindo os Transtornos Mentais Relacionados ao Trabalho (TMRT). Mesmo que os dados do TMRT não consigam mensurar com precisão a magnitude dos casos, evidenciam sua relevância em relação ao adoecimento dos trabalhadores.

Analisando o período de 2006 a 2017, no Sistema de Informação de Notificação de Agravos do Sistema Único de Saúde (SINAN), foram registrados 8.474 casos de transtornos mentais relacionados ao trabalho no Brasil (SINAN, 2019). Paralelo a isso, considera-se o trabalho um importante fator de adoecimento, de desencadeamento e de crescente aumento de distúrbios psíquicos. A debilidade social do 
trabalho gera processos complexos, que associados a condições precárias de trabalho e emprego, geram insegurança e vulnerabilidade (ARAÚJO et al.,2017).

Desta forma, o processo de trabalho engloba fatores objetivos e subjetivos. Os fatores subjetivos estão relacionados a forma de trabalho e o gasto produtivo das energias vitais, que são usados para a satisfação de suas necessidades. Os fatores objetivos referem-se aos objetos (matéria-prima) e o meio. Conjectura-se que o trabalho é responsável por mediar entre aquilo que se busca enquanto sujeito, e por aquilo que mantêm a sua sobrevivência (CANTARELLI et al, 2017). Os aspectos relacionados a condições organizacionais, contexto organizacional, ambiente de trabalho, somados às características pessoais, são elementos responsáveis pelo adoecimento do trabalhador (ARAÚJO et a., 2017).

A Rede Nacional de Atenção Integral à Saúde do Trabalhador (RENATS), criado através da Portaria n 1.679 de 2002, foi organizado e estruturado por: ações na rede de atenção básica e no Programa de Saúde da Família (PSF), Rede de Centros de Referência em Saúde do Trabalhador (Cerest) e ações na rede assistencial de alta e média complexidade.

O Centro de Referência em Saúde do Trabalhador (CEREST), é uma estratégia da Rede Nacional de Atenção Integral à Saúde do Trabalhador (RENATS), que visa a promoção de saúde, com a proposta de reduzir a incidência de morbimortalidade da população trabalhadora (Portaria de Consolidação n.2, de 28/09/2017, Anexo XV). Os CEREST devem articular-se como polos irradiadores (em seu território de ação) em observância à cultura que perpassa a relação de trabalho, que pode culminar tanto em produção de saúde como em doença, tendo função de suporte técnico e científico da saúde do trabalhador. Deve atuar articulando-se também com toda a rede do SUS, de modo que os agravos decorrentes do trabalho sejam atendidos nos diferentes níveis de atenção do SUS (CARDOSO; ARAÚJO, 2016).

Santana e Neves (2017), ao discorrerem acerca da rede de atenção integral à saúde do trabalhador, analisa que entre a implantação dessa, no ano de 2002, e a publicação da política nacional de saúde do trabalhador, em 2012, a saúde do trabalhador (ST) modifica o eixo, no sentido de que, se, no início, os CEREST tinham caráter de assistência ambulatorial, a datar de 2005, com a Portaria 2.437, passa-se então a tomar a vigilância epidemiológica e sanitária como foco organizativo, por meio das definições vindas da Rede Sentinela e da atenção primária, e com a política nacional de saúde do trabalhador, devem assumir o apoio matricial de toda a Rede SUS, em especial à atenção primária e dela à Estratégia de Saúde da Família. Uma forma de identificar demandas que necessitam de ação é por meio do estudo epidemiológico local (SANTANA; NEVES, 2017).

No âmbito da Saúde do Trabalhador, notificações compulsórias estão relacionadas a agravos, doenças ou eventos, por consequência de trabalho. Em 2014, a Portaria ${ }^{\circ} 1.271$, juntamente com a Portaria $\mathrm{n}^{\circ} 1.984$ mantém como notificação compulsória os transtornos mentais decorrentes de atividade laboral, condensando as demais doenças em outras cinco proposições: câncer relacionado ao trabalho; dermatoses 
ocupacionais; lesões por esforços repetitivos/distúrbios osteomusculares relacionados ao trabalho (LER/ DORT); perda auditiva induzida por ruído (PAIR) relacionada ao trabalho e pneumoconioses relacionadas ao trabalho. Dentre as doenças/transtornos citados, alguns deles, como os transtornos mentais decorrentes de atividade laboral e as LER/DORT, são frequentemente relatados em estudos que investigam o adoecimento docente (NETO, 2018).

O SUS é reflexo de um conjunto de embates políticos e ideológicos, travado por diferentes protagonistas ao longo dos anos, não sendo fruto de um cenário atual, mas sim marcado por uma longa trajetória de lutas e formulações (PAIVA; TEIXEIRA, 2014). Em todo o país, o SUS deve ter a mesma doutrina e forma de organização, definido na Constituição como um conjunto de elementos doutrinários e de organização com princípios da universalização, da equidade, da integralidade, da descentralização e da participação popular (BRASIL, 2002).

Segundo a legislação brasileira, Lei no 8.080/90, a saúde é um direito fundamental do ser humano, cabendo ao poder público - União, Estados, Distrito Federal e Municípios - garantir este direito, através de políticas sociais e econômicas que visem à redução dos riscos de se adoecer e morrer, bem como, o acesso universal e igualitário às ações e serviços de promoção, proteção e recuperação da saúde.

Além da saúde pública estar voltada ao controle do contágio do coronavirus, há uma enorme preocupação com o que vai exceder ao que se refere a saúde mental neste momento de isolamento e distanciamento social. Desta forma, como garantir que a vida - considerando aspectos psicossociais seguirá seu curso normal, ao tratar de um vírus que não oferece saídas simplórias e fáceis? Devem ser levados em consideração, além do isolamento, aspectos, como, por exemplo, a diminuição da renda familiar, o desemprego, a falta de informação e/ou excesso de informações negativas, podem levar o sujeito a desenvolver ou agravar transtornos mentais (DUARTE et al., 2020).

Vendo que muitos profissionais estão atuando na linha de frente durante a pandemia da COVID-19, é imprescindível monitorar fatores nocivos à saúde dos mesmos. Este artigo tem a finalidade de narrar as experiências e estratégias utilizadas pelo CEREST, localizado no noroeste do Estado do Rio Grande do Sul, no período de julho a setembro de 2020. Além disso, discutir aspectos relacionadas a saúde dos trabalhadores que estão atuando durante a pandemia

Trata-se de um relato de experiência de estágio realizado no CEREST Fronteira Noroeste, no qual foram realizados encontros semanais com grupos de profissionais da área da saúde. Os grupos foram conduzidos por duas estagiárias de psicologia do décimo semestre da Sociedade Educacional de Três de Maio - SETREM. Neste relato de experiência, destacam-se aspectos relacionados à saúde mental do profissional da saúde, utilizando ferramentas com base na Terapia Cognitivo Comportamental (TCC) e o psicodrama. O conteúdo de cada encontro foi transcrito e analisado qualitativamente, identificando particularidades em suas falas, bem como, o que circunda em cada um dos grupos. Vale ressaltar ainda que 
foram respeitados nesta experiência aspectos éticos preconizados pela Resolução 466 de 2012 do Conselho Nacional de Ética na Pesquisa - CONEP.

\section{RESULTADOS E DISCUSSÕES}

No mês de julho de 2020 o CEREST Fronteira Noroeste, juntamente com um grupo de estagiários de psicologia do décimo semestre da instituição SETREM - Sociedade Educacional de Três de Maio - RS, iniciou um trabalho de cuidados em saúde mental aos trabalhadores de saúde que estão trabalhando no SUS no município de Santa Rosa/RS.

Foram planejadas ações em saúde mental que abarcasse todos os setores da FUMSSAR, desde os trabalhadores que atuam no setor de transporte, estoque, compras, contabilidade, técnicos de informática (TI), administrativo, até os profissionais de saúde que estão atuando na linha de frente no enfrentamento a pandemia da COVID-19 como os técnicos de enfermagem, enfermeiros, nutricionistas, psicólogos, assistentes sociais, terapeutas ocupacionais, fonoaudiólogos, fisioterapeutas, farmacêuticos, médicos, odontólogos, agentes comunitárias de saúde, agentes de combate às endemias, serviços gerais, etc.

As atividades grupais ocorreram em diferentes momentos com cada setor da Fundação Municipal de Saúde de Santa Rosa, onde aconteceram três encontros com a temática da saúde mental durante a pandemia da covid-19, com o objetivo de fornecer um espaço de fala para estes profissionais e principalmente dialogar e orientar os mesmos sobre a caracterização das relações entre saúde mental e as suas ocupações, além disso pensar juntamente com as equipes diferentes formas de enfrentamento em saúde mental durante a pandemia do Covid-19. As atividades de grupo eram planejadas para que ocorressem dentro de um tempo máximo de 30 a 40 minutos por conta da demanda de trabalho acrescida neste período de pandemia.

Para a realização das atividades as estagiárias utilizaram-se de conceitos e pressupostos básicos da abordagem e teoria cognitivo-comportamental, pois a mesma vem demonstrando alta eficácia em pesquisas científicas e tratamento de transtornos psicológicos. A Terapia Cognitivo Comportamental criada na década de 1960, por Aaron Beck, é uma abordagem focada no aqui e agora, diretiva e ficada. Utiliza-se do conceito da estrutura biopsicossocial - fatores biológicos, psicológicos e sociais - na determinação e compreensão dos fenômenos relativos à psicologia humana (BARROS et al., 2014).

Os grupos terapêuticos são utilizados em diversos contextos e com diferentes abordagens, coordenados muitas vezes por profissionais de equipes interdisciplinar. Parte-se do pressuposto de que as ações promotoras de Saúde Mental, precisam incidir não só sobre o sujeito, mas sobre todos seus vínculos intersubjetivos (CARNEIRO et al, 2010). Neste sentido, o grupo torna-se um espaço terapêutico para os pacientes e de afirmação de uma nova atuação dos profissionais. O trabalho com grupos se constitui um 
dos principais recursos, principalmente no campo da saúde mental e assistência a saúde. (SOUZA, et al, 2004).

No grupo enfoca-se as trocas de experiência, o diálogo entre os membros e as mudanças que isso gera na percepção de cada um. É através do convívio entre eles que surgem os debates acerca das práticas do cuidado, e é nesses encontros que os meios para a resolução dos problemas coletivos acontecem, buscando alternativas e apoio emocional para sua superação (HEBERLE; OLIVEIRA, 2016).

Inicialmente pedia-se para que cada participante do grupo se apresentasse, falando o seu nome, idade, local de trabalho e como tem se sentido nos últimos meses com o decorrer da pandemia. Posteriormente, utilizando-se de uma folha de papel e caneta solicitava-se para que cada participante colocasse em forma de um gráfico ilustrativo as áreas da sua vida, como trabalho, família, lazer, saúde e convívio social, e descrevessem como se sentiam em cada área da sua vida e como a pandemia havia afetado a cada uma dessas esferas. Através destas duas atividades conseguimos fazer um breve levantamento de demandas a serem trabalhadas diferentemente com cada setor de trabalho.

No primeiro encontro os trabalhadores da saúde em sua maioria relataram que o trabalho tem exigido mais atenção e cuidados neste momento por estarem atuando na linha de frente ao combate do coronavírus, relataram igualmente sentirem-se sobrecarregados, cansados, estressados, ansiosos e temerosos em relação ao futuro, sobretudo em relação a uma possível contaminação e contágio para os familiares. Percebeu-se que a relação com os colegas de trabalho também acaba sendo afetada muitas vezes por conta desses estressores. A relação com a família, como filhos, esposo(a), pais e avós têm se estreitado por estarem em contato continuamente por conta do isolamento social, fechamento de locais de trabalhos, fechamento de escolas, creches e universidades, porém também ocorreu o distanciamento de alguns parentes que anteriormente eram próximos e círculo de amigos. Os gráficos ilustrados pelos trabalhadores demonstraram que o convívio social com amigos, a realização de atividades e hobbies quase que se anularam neste período por conta do distanciamento social.

Percebeu-se que há algumas equipes que não atuam diretamente com o público ou pacientes do Covid-19, mas que atuam em áreas de suporte e assistência a esses trabalhadores, neste caso pode-se perceber uma dinâmica diferenciada. Ambos relataram que o trabalho, tem exigido mais atenção e cuidados neste momento por estarem passando pela pandemia, relataram igualmente sentir acrescido às demandas de trabalho, mas diferentemente dos grupos anteriores estes grupos não relataram sentimentos como medo, ansiedade, estresse, etc. Estes grupos relataram estar cientes das problemáticas advindas da pandemia, mas não temem uma possível contaminação e apresentam-se esperançosos em relação ao futuro.

No segundo encontro a atividade proposta pelas estagiárias foi para dialogar sobre o reconhecimento de nossos sentimentos e emoções e a comunicação da equipe no local de trabalho. Para isso, havia dentro de uma caixa algumas palavras como medo, ansiedade, tristeza, isolamento, angústia, 
resiliência, perseverança, coragem, etc. Esta caixa era deixada em cima de uma mesa para que de um a um os trabalhadores retirassem um papel da caixa e falasse sobre aquela emoção ou sentimentos descritos no papel. Com essa dinâmica os trabalhadores conseguiram perceber e colocar em palavras quais os sentimentos que estão presentes atualmente no seu dia a dia, puderam refletir sobre como os mesmos têm lidado com essas problemáticas e além disso, através do diálogo puderam observar que não só eles como muitos colegas estavam passando pela mesma situação e assim ter mais paciência, colaboração e empatia com a dor do outro.

No terceiro e último encontro as estagiárias trouxeram alguns slides para dialogar sobre as formas de enfrentamento e hábitos que podem auxiliar na diminuição dos impactos negativos à saúde mental advindos da pandemia. Nesses slides continham informações retiradas de uma cartilha da Fiocruz; denominada de “As estratégias de cuidado psíquico recomendadas em situação de pandemia”. Contudo, foram-lhes apresentadas as seguintes estratégias: a importância de estabelecer uma nova rotina diária e adaptar-se às novas condições associadas a pandemia, formas de gerenciar o fluxo constante de informações sobre o Covid-19, apresentação de fontes confiáveis de busca e como evitar as informações falsas geradoras de pânico, a importância da prática de exercícios ou práticas de respiração para gerenciar o estresse e a ansiedade, como manter uma alimentação saudável e quais os benefícios de uma boa noite de sono, meios para buscar ou manter boas conexões interpessoais, ser gentil e realista consigo mesmo nestes momentos. Além disso, abordamos questões relacionadas ao uso do cigarro, álcool e outras drogas, que muitas vezes são usadas para lidar com as emoções. Além disso foram utilizadas outras dinâmicas psicodramáticas, para a interação do grupo e técnicas de relaxamento corporal e autopercepção.

Todos os grupos trouxeram que se beneficiaram de alguma forma com estes encontros, pois muitas vezes com a correria do dia a dia, não tiram um momento para sentir seu corpo, sua respiração e muito menos para refletir sobre seus pensamentos, emoções e consequentemente como isso tem afetado seu comportamento e a sua rotina. Considera-se, portanto, que trabalhos de diálogo e prevenção em saúde mental são tão necessários quanto cuidar da saúde física neste momento de pandemia.

\section{CONSIDERAÇÕES FINAIS}

Diante da realidade que estamos enfrentando onde há ausência da disponibilização de vacinas ou de um tratamento comprovadamente eficaz para o tratamento da Covid-19, a estratégia de distanciamento social ainda tem se mostrado a maneira mais eficiente de controlar e amenizar os danos da doença. Entretanto, as equipes de assistência à saúde e os profissionais que estão no cuidado direto com o público de pacientes com suspeita ou diagnosticados com a Covid-19, estão expostos a contaminação, por estarem envolvidos direta e indiretamente no enfrentamento da pandemia e estarem cotidianamente em risco de adoecer pelo coronavírus. (TEIXEIRA et al., 2020). Além do adoecimento físico, esta exposição pode vir 
a gerar manifestações psicopatológica, caso não seja feita nenhuma intervenção de cuidado específico para as reações e sintomas manifestados (NOAL et al., 2020)

A partir desta prática de estágio, verificou-se a necessidade de proporcionar um espaço de fala a esses profissionais, bem como uma escuta acolhedora aos sentimentos e preocupações desses indivíduos. Tendo em vista a criação de um vínculo respeitoso e de suporte para minimizar as necessidades urgentes levantadas no grupo. Orientar técnicas que podem reduzir o estresse, ansiedade e as comorbidades advindas desta problemática, além de auxílio com questões práticas. As intervenções são no sentido de respeitar o momento que estamos enfrentando, fortalecer o senso de resiliência e interconexão para uma melhor adaptação à nova realidade. 


\section{REFERÊNCIAS}

ARAÚJO, T. M. et al. Vigilância em Saúde Mental e Trabalho no Brasil: características, dificuldades e desafios. Revista Ciência e Saúde Coletiva, 22(10), 3235-3246, 2017.

BARROS, J. R. F. A Terapia Cognitivo Comportamental no tratamento de pacientes com dor crônica. Revista Ciência Biológicas e da Saúde, 2(2), p.77-90, 2014.

BRASIL. Ministério da Saúde. Sistema Público de Saúde. Brasília-DF, 2002. Recuperado em https://bvsms.saude.gov.br/bvs/publicacoes/sistema_saude.pdf Acesso em 01 de setembro de 2020.

BRASIL Ministério da Saúde. A epidemiologia da Saúde do Trabalhador no Brasil. Brasília-DF,2020. Recuperado em https://www.saude.gov.br/images/pdf/2020/April/27/epidemiologia-saude-trabalhadorufba-ms-4mar20-isbn.pdf Acesso em 01 de setembro de 2020.

CANTARELLI, A. G. ; FACCI. M. G. D. ; CAMPOS, H. R. Trabalho docente e personalidade: alienação e adoecimento. In: Marilda Gonçalves Dias Facci; Sônia da Cunha Urt. (Org.). Precarização do trabalho, adoecimento e sofrimento do professor.Teresina: EDUFPI, 2017,v. 01, p. 19-44.

CARDOSO, M. C.B; ARAÚJO, T. M. Os Centros de Referência em Saúde do Trabalhador e as ações em saúde mental: um inquérito no Brasil. Revista Brasileira Saúde Ocupacional, 41(7), 2016.

CARNEIRO, A. C.et al. Educação Popular em Saúde Mental: relato de uma experiência. Revista Saúde Sociedade, 19(2), p 462-474, 2010.

Conselho Nacional de Secretário de Saúde - CONASS. A Atenção Primária e as Redes de Atenção à Saúde. Brasília, 2015. Recuperado em https://www.conass.org.br/biblioteca/pdf/A-Atencao-Primaria-e-as-Redesde-Atencao-a-Saude.pdf

DUARTE, M. Q. et al. COVID-19 e os impactos na saúde mental: uma amostra do Rio Grande do Sul, Brasil. Revista Ciência e Saúde Coletiva, 25(9),2020.

Fundação Municipal da Saúde de Santa Rosa - FUMSSAR. Plano Municipal de Saúde de Santa Rosa. 2013. http://www.fumssar.com.br/wpcontent/uploads/2014/05/Plano_Municipal_de_Sa\%C3\%BAde_2014-2017_Santa-Rosa.pdf

NOAL, D.S. et al. Saúde mental e Atenção Psicossocial na Pandemia Covid 19 Recomendações gerais. Fundação Oswaldo Cruz, 2020. Disponivel em: $\underline{\text { http://profsaude- }}$ abrasco.fiocruz.br/sites/default/files/publicacoes/cartilha_recomendacoes_gerais_06_04.pdf

MAEYAMA, et al. Atenção Básica à Saúde: aproximando teoria e prática. Itajaí: UNIVALI, 2018. Disponível em:https://www.univali.br/vida-no-campus/editora-univali/ebooks/Documents/ecs/Aten\%C3\%A7\%C3\%A3o\%20B\%C3\%A1sica\%20\%C3\%A0\%20Sa\%C3\%BAde\% 20Aproximando\%20Teoria\%20e\%20Pr\%C3\%A1tica.pdf

MATTEI, L. A crise econômica decorrente da covid-19 e as ações da equipe econômica do governo atual. NECAT - Núcleo de Estudos de Economia Catarinense. Disponível em: https://noticias.paginas.ufsc.br/files/2020/03/31.03.20-TD-NECAT-035-2020.pdf 
MS-Brasil, Ministério da Saúde do Brasil. (2020). Influência da COVID-19 na Saúde Mental de Profissionais de Saúde Survey. Secretaria de Gestão Do Trabalho e Da Educação Em Saúde (SGTES) e Associação Brasileira de Psiquiatria (ABP). Disponível em: https://pt.surveymonkey.com/r/Covid19_SaudeMental_SGTES

NETO, A. G. C. A subnotificação de Doenças e agravos à Saúde dos Profissionais da Educação: uma análise do caso do magistério público do Distrito Federal. Revista Gestão Universitária, v.10, 2018.

SINAN: Sistema de Informação de Agravos de Notificação. 2018. Disponível em http://docs.wixstatic.com/ugd/303ec7_e13f0b75d9ef4d98a8cab8132e68cfaf.pdf. Acesso em 10 de setembro de 2020.

PAIVA, C. H. A; TEIXEIRA, L. A. Reforma sanitária e a criação do Sistema Único de Saúde: notas sobre contextos e autores. Revista História, Ciência, Saúde, 21(1), p.15-35, 2014.

PORTARIA DE CONSOLIDAÇÃO No 2, DE 28 DE SETEMBRO DE 2017. Disponível em http://bvsms.saude.gov.br/bvs/saudelegis/gm/2017/prc0002_03_10_2017.html

SCHMIDT, B., et al. Impactos na Saúde Mental e Intervenções Psicológicas Diante da Pandemia do Novo Coronavírus (COVID-19). Revista Estudos de Psicologia, v.37, 2020.

SOUZA, A. M. A. et al. Grupo terapêutico: sistematização da assistência de enfermagem em saúde mental. Revista Texto Contexto Enferm, 13(4), p. 625-32,2004.

TEIXEIRA, C. F. S. et al. A saúde dos profissionais de saúde no enfrentamento da pandemia de Covid-19. Revista Ciência \& Saúde Coletiva, 25(9), p.3465-347, 2020. 